\title{
A Constitutive Model for Molecular Weight and Concentration Effects in Polymer Blends
}

\author{
Donald C. Bogue, ${ }^{*}$ Toshiro Masuda, ${ }^{* *}$ Yoshiyuki Einaga, ${ }^{* * *}$ \\ and Shigeharu ONOGI ${ }^{* *}$ \\ Department of Polymer Chemistry, Kyoto University, Kyoto, Japan.
}

(Received June 25, 1970)

\begin{abstract}
Recent work with binary blends of narrow-distribution polystyrenes has shown that the elastic properties depend on the molecular weight distribution in a complex way when the molecular weights are both above the entanglement point. In particular the steady-state compliance $J_{e}{ }^{0}$ becomes proportional to $w_{2}{ }^{-2}$ when $w_{2}$, the weight fraction of the higher molecular-weight species, approaches unity. These results led to the suggestion that a high-order (nonlinear) blending law might be required above the entanglement point.

In the present work the general problem of modelling the molecular weight dependence in the transition, rubbery plateau, and terminal flow regimes is discussed, including models for the juncture points between these regions. A general mathematical framework is presented which includes the Rouse spectrum and a linear blending law for low molecular weights (and also for the short-time portion of the spectrum generally); a network spectrum and a quadratic blending law for the higher molecular weights; and a mixture of these models for intermediate cases. Also, results for wide-distribution mixtures and for $n$-th order blending laws are stated.

KEY WORDS Binary Blend / Blending Law / Molecular Weight

Distribution / Viscoelasticity / Relaxation Spectrum / Steady-State

Compliance / Viscosity /
\end{abstract}

As is well-known from many studies with bulk polymers, the dependence of the zero-shear viscosity on molecular weight varies from a linear one at low molecular weight to a dependence on approximately the 3.5 power at high molecular weight. ${ }^{1,2}$ The transition point between these two regions is referred to as the critical molecular weight, $M_{c}$, and also marks the beginning of significant non-Newtonian effects at high shear rates. The recent work of $\mathrm{Akovali}^{3}$ and Masuda, et al., ${ }^{4}$ on blends of narrow-distribution polystyrenes has made clear many of the effects of molecular weight and molecular weight distribution. In the case of a single molecular weight, $M$, Onogi and coworkers ${ }^{5}$

* Presently on leave from the Department of Chemical and Metallurgical Engineering, University of Tennessee, Knoxville, Tennessee, U.S.A.

** To whom correspondence should be addressed.

*** Department of Industrial Chemistry, Kyoto University, Kyoto, Japan. observe dependence of the viscosity on $M^{1}$ and $M^{3.7}$, as expected, and dependence of an elastic coefficient (see eq 3) on $M^{3}$ and $M^{7.5}$, below and above $M_{c}$, respectively. In the case of binary blends, they continue to observe the first power and 3.7 power dependence of viscosity, if now a weight-average molecular weight, $\bar{M}$, is used; but for the elastic properties, more complicated effects are observed. For example, the steady-state compliance depends significantly on the weight fractions and, in the entanglement region, is proportional to $w_{2}^{-2}$ when $w_{2}$, the weight fraction of the higher molecular-weight species, is close to unity. It is the purpose of the present work to attempt a phenomenological summary of these diverse effects.

\section{STATEMENT OF THE MODEL (SINGLE REGIME DOMINATING)}

We will use here a linear integral Maxwell model given by $^{6}$ 


$$
\begin{aligned}
\sigma_{i j}= & -p \delta_{i j}+\int_{0}^{\infty}\left[\int_{-\infty}^{+\infty} \frac{H(\tau)}{\tau} e^{-\left(t-t^{\prime}\right) / \tau}\right. \\
& \times \mathrm{d} \ln \tau] \varepsilon_{i j} d\left(t-t^{\prime}\right)
\end{aligned}
$$

where $\sigma_{i j}$ is the stress tensor, $p$ is an arbitrary scalar stress, $\delta_{i j}$ is the Kronecker delta, $\varepsilon_{i j}$ is a linear form of the Finger strain tensor, $t-t^{\prime}$ is the difference between the present time and some arbitrary past time and $H(\tau)$ is the relaxation spectrum. For simple shear with $\dot{\gamma}$ as the shear rate and for oscillatory flows with $\omega$ as the angular frequency, this model gives the following results

$$
\begin{gathered}
\eta_{0}=\lim _{\dot{\gamma} \rightarrow 0} \frac{\sigma_{12}}{\dot{\gamma}}=\lim _{\omega \rightarrow 0} \frac{G^{\prime \prime}(\omega)}{\omega}=\int_{0}^{\infty} H(\tau) d \tau \\
\theta_{0}=\lim _{\dot{\gamma} \rightarrow 0} \frac{\sigma_{11}-\sigma_{22}}{2 \dot{\gamma}^{2}}=\lim _{\omega \rightarrow 0} \frac{G^{\prime}(\omega)}{\omega^{2}}=\int_{0}^{\infty} \tau H(\tau) d \tau
\end{gathered}
$$

where $\eta_{0}$ is the zero-shear viscosity and $\theta_{0}$ is the zero-shear elastic coefficient and $G^{\prime \prime}(\omega)$ and $G^{\prime}(\omega)$ are respectively, the loss and storage moduli. We will also define a steady-state compliance $J_{e}^{0}$ by the equation ${ }^{7}$

$$
J_{e}{ }^{0}=\frac{\theta_{0}}{\eta_{0}{ }^{2}}=\frac{\int_{0}^{\infty} \tau H(\tau) d \tau}{\left[\int_{0}^{\infty} H(\tau) d \tau\right]^{2}}
$$

It is very instructive to consider the predictions of a one-term discrete Maxwell element, while recognizing, of course, that one element cannot reflect the detailed mechanisms shown by the relaxation spectrum. If $G$ is the elastic modulus and $\tau$ is the time constant in the Maxwell element, we have the following predictions

$$
\eta_{0}=G \tau, \quad \theta_{0}=G \tau^{2}, \quad J_{e}^{0}=\frac{1}{G}
$$

It is a very fundamental idea in rheological equations that one must model separately the two constants $G$ and $\tau$ (or their counterparts in an equation for the spectrum). This idea has been carefully followed in the time-temperature superposition principle first proposed by Leaderman $^{8}$ and the same separation needs to be modelling molecular weight effects.

From the molecular theories one may make certain observations about the parameters $G$ and $\tau$. We do not imply, of course, that the theories use a single Maxwell element, but this simple framework allows one to make several useful comparisons. Two very basic ideas are invloved. On the one hand, in the Rouse dilutesolution theory ${ }^{9}$ and in the extension of this theory to undiluted polymers of low molecular weight, ${ }^{10}$ the results for a single molecular-weight species $M$ are of the form

$$
G=\text { const } / M, \quad \tau=\text { const } \cdot M^{2}
$$

The dilute-solution theory of $\mathrm{Zimm}^{11}$ is of essentially the same form but with the exponent in the time-constant equation being 1.5 instead of 2. The so-called modified Rouse theory noted above also has been applied above the entanglement point but we believe the network theories are more suitable there. The dependence of the modulus on the inverse of the molecular weight is physically related to the number of free ends in the system. In contrast the theories of Lodge, ${ }^{12}$ Yamamoto $^{13}$ and Hayashi ${ }^{14}$ consider a network structure, the modulus of which depends on the number of junction points in the network, but the effect of molecular weight is either not considered or is absent. As far as molecular weight effects are concerned the network concept may be summarized by

$$
G=\mathrm{const}
$$

The molecular weight dependence of the time constant is dealt with explicitly in the Hayashi theory $^{14}$ and experimentally in a number of studies $^{3,15}$ and is of the form

$$
\tau=\text { const } \cdot M^{3.5}
$$

Some variation in the exponent has been reported.

If we insert, then, eq 6,7 and 8 into eq 5 , we obtain the following predictions for undiluted polymers:

Low molecular weight (Rouse):

$\eta_{0}=$ const $\cdot M, \theta_{0}=$ const $\cdot M^{3}, J_{e}{ }^{0}=$ const $\cdot M$

High molecular weight (network):

$\eta_{0}=$ const $\cdot M^{3.5}, \theta_{0}=$ const $\cdot M^{7.0}$

$J_{e}^{0}=$ const

Except for some small variations in the exponents, these results are in accord with those cited 


\section{A Constitutive Model for Molecular Weight and Concentration Effects in Polymer Blends}

in the opening paragraphs.

The question now becomes one of considering the entire relaxation spectrum and the relationship of the characteristic modulus (or moduli) and time constant (or constants) to the molecular weight. And also of considering the appropriate concentration dependence when different molecular-weight species are mixed. For blends Ninomiya ${ }^{16}$ and Ninomiya and Ferry ${ }^{17}$ have proposed a linear blending law for the elastic modulus, in the spirit of the Rouse model, which is equivalent to

$$
H_{b}(\tau)=v_{1} H_{1}\left(\frac{\tau}{\lambda_{1}}\right)+v_{2} H_{2}\left(\frac{\tau}{\lambda_{2}}\right)
$$

where $H_{b}(\tau)$ is the relaxation spectrum of the mixture, the $v_{i}$ are the volume fractions, the $H_{i}$ are the spectra for the pure species, and the $\lambda_{i}$ are dimensionless shift factors which depend on the molecular weight. In most studies the differences between volume fractions and weight fractions are small ones and in the subsequent discussion we will use the more convenient weight fractions $w_{1}$ and $w_{2}$. The model of eq 10 predicts many of the desired properties, as, for example, a dependence of the compliance $J_{e}^{0}$ on composition such that the compliance for the mixture is higher than that of either of the pure constituents. Recent work with high molecular-weight systems has shown, however, that the strong concentration dependence of the compliance (proportional to $w_{2}^{-2}$ as $w_{2} \rightarrow 1$ ) is not in agreement with the inverse prediction $\left(w_{2}^{-1}\right)$ which comes from eq 10 . Also inspection of the dynamic response curves shows various complicated couplings between the two species which cannot be explained by linear blending rules.

To derive the viscosity and elastic coefficients from eq 10 requires an implicit assumption about the molecular weight dependence of the limits in eq 2 and 3; or otherwise stated, if the integration is carried out from zero to infinity, one must assume that the shift factors $\lambda_{1}$ and $\lambda_{2}$ are independent of the time scale. This can only be true, of course, when only one regime (such as the the terminal flow regime) dominates in the integration. Because we wish to deal with the problem more generally, we will use a modified notation in which shift factors as such are not introduced but in which the molecular weight dependence of the spectrum and also the limits are stated explicitly.

In the new notation, then, the linear weightaverage mixing law is given by

$$
H_{b}=w_{1} H_{1}+w_{2} H_{2}
$$

To explain the complex effects in high molecularweight systems, Masuda, et al., ${ }^{4}$ have suggested that a quadratic mixing law might be more appropriate

$$
H_{b}=w_{1}^{2} H_{11}+2 w_{1} w_{2} H_{12}+w_{2}^{2} H_{22}
$$

where $H_{11}$ and $H_{22}$ are the relaxation spectrum of the pure species and $H_{12}$ is a cross-relaxation spectrum. This equation has considerable intuitive appeal in that cross term reflects entanglements between different molecular-weight species. The idea of quadratic interaction has some basis in the Fixman equation for solutions as used by Williams ${ }^{18}$ which is of the form

$$
\sigma_{i j}=\sigma_{i j}^{0}+n F_{i j}+n^{2} G_{i j}
$$

where $\sigma_{i j}^{0}$ is the stress due to the solvent and external isotropic pressure, $n$ is the number of molecules per unit volume, $F_{i j}$ is an intramolecular potential term and $G_{i j}$ is an intermolecular (interaction) potential term. Certain assumptions about the potential functions must be made to produce eq 13, which is a truncated form that could contain higher-order terms also. We do not appeal to eq 13, however, except in a qualitative way. Later eq 12 will be generalized to permit a skewed weighting of the species 1 and 2 .

In cases where a single regime dominates, we will assume as did Ninomiya and Ferry that the molecular weight dependence of the limits is the same as that of the spectrum. As to the form of the spectra, the basic philosophy will be that the noninteracting form (eq 6) and a linear mixing rule (eq 11) are appropriate where all molecular-weight species are below $M_{c}$; and that the network form (eq 7 and 8) and the quadratic mixing rule (eq 12) are appropriate where all species are above $M_{c}$. We will later generalize the results for mixtures of low and high molecular weight species. Finally the requirement that the viscosity of the blend depends only on the weight-average molecular 
weight places a strong restriction on the form that the quadratic spectrum may take and requires that it be proportional to $M_{i} M_{j}$. All of these considerations lead to the results ${ }^{\dagger}$ :

For $M_{i}<M_{c}$ :

$$
H_{i}(\tau)=\frac{G_{c}{ }^{\prime} / M_{i}{ }^{*}}{f_{i}\left(\tau / \tau_{i}(M)\right)} \quad\left[\text { for } a_{i} \tau_{i}(M)<\tau<b_{i} \tau_{i}(M)\right]
$$

where $\tau_{i}(M)=\tau_{c}{ }^{\prime} M_{i}{ }^{2}$

For $M_{i}>M_{c}$ :

$$
\begin{aligned}
H_{i j}(\tau)= & \frac{G_{c}{ }^{\prime \prime \prime}}{g_{i j}\left(\tau / \tau_{i j}(M)\right)} \quad \text { for } a_{i j} \tau_{i j}(M) \\
& \left.<\tau<b_{i j} \tau_{i j}(M)\right]
\end{aligned}
$$

where $\tau_{i j}(M)=\tau_{c}{ }^{\prime \prime \prime} M_{i} * M_{j} * \bar{M}^{* 1.5}$

The notation $\tau_{i}(M)$ and $\tau_{i j}(M)$ is to indicate that these characteristic time constants are dependent on the molecular weight (or molecular weight distribution). All other quantities are characteristic constants for the material and independent of molecular weight and concentration. The symbol $M_{i}^{*}=M_{i} / M_{c}$ is the ratio of the molecular weight of species " $i$ " to the critical molecular weight and the average $\bar{M}^{*}$ is given by

$$
\bar{M}^{*}=w_{1} M_{1}^{*}+w_{2} M_{2}{ }^{*}
$$

\section{PREDICTIONS OF MODEL (SINGLE REGIME DOMINATING)}

We will now write out explicitly the predictions for the rheological properties when one regime dominates. Using eq 11 and 12 and the postulates of eq 14 and 15 we obtain from eq 2 the following results for the viscosity.

For $M_{i}^{*}<1$ :

$$
\eta_{0}=\frac{G_{c}^{\prime} \tau_{c}^{\prime}}{M_{c}}\left\{w_{1} M_{1} \int_{a_{1}}^{b_{1}} \frac{d \xi}{f_{1}(\xi)}+w_{2} M_{2} \int_{a_{2}}^{b_{2}} \frac{d \xi}{f_{2}(\xi)}\right\}
$$

For $M_{i}^{*}>1$ :

$$
\eta_{0}=\frac{{G_{2}}^{\prime \prime \prime} \tau_{c}{ }^{\prime \prime \prime}}{M_{c}^{3.5}} \bar{M}^{1.5}\left\{w_{1}{ }^{2} \bar{M}_{1}^{2} \int_{a_{11}}^{b_{11}} \frac{d \xi}{g_{11}(\xi)}\right.
$$

† It is well to state explicitly that the equations for $H_{i}$ and $H_{i j}$ are abbreviated index notation but not tensor equations. The only tensor equations in the paper are eq 1 and 13.

$$
\left.+2 w_{1} w_{2} M_{1} M_{2} \int_{a_{12}}^{b_{12}} \frac{d \xi}{g_{12}(\xi)}+w_{2}{ }^{2} M_{2}^{2} \int_{a_{22}}^{b_{22}} \frac{d \xi}{g_{22}(\xi)}\right\}
$$

Because we are dealing with a single regime we will say that the dimensionless shape integrals are equal

$$
\begin{gathered}
K_{0}=\int_{a_{1}}^{b_{1}} \frac{d \xi}{f_{1}(\xi)}=\int_{a_{2}}^{b_{2}} \frac{d \xi}{f_{2}(\xi)} \\
K_{1}=\int_{a_{11}}^{b_{11}} \frac{d \xi}{g_{11}(\xi)}=\int_{a_{12}}^{b_{12}} \frac{d \xi}{g_{12}(\xi)}=\int_{a_{22}}^{b_{22}} \frac{d \xi}{g_{22}(\xi)}
\end{gathered}
$$

We then obtain the following results, which are independent of the shape of the functions $f$ or $g$; these results incorporate, for example, the Rouse prediction of $f^{1 / 2}$ and higher slopes in the entanglement region. Eq 17 and 18 then reduce to:

For $M_{1}^{*}<1$ :

$$
\eta_{0}=G_{c}{ }^{\prime} \tau_{c}{ }^{\prime} K_{0}\left(\frac{\bar{M}}{M_{c}}\right)
$$

For $M_{i}^{*}>1$ :

$$
\eta_{0}=G_{c}{ }^{\prime \prime \prime} \tau_{c}{ }^{\prime \prime \prime} K_{1}\left(\frac{\bar{M}}{M_{c}}\right)^{3.5}
$$

The validity of these results is shown in

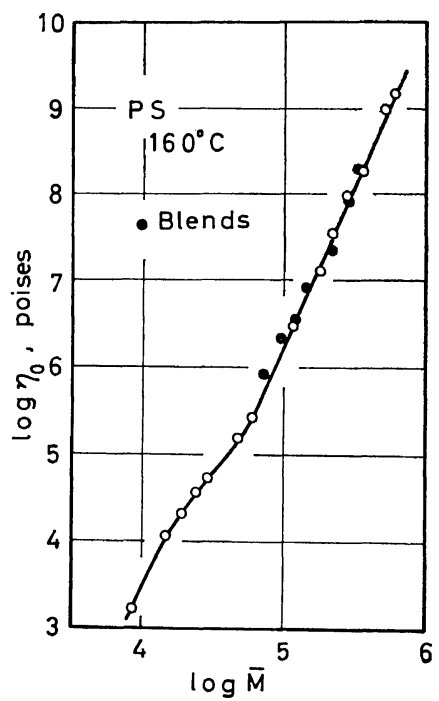

Figure 1. Zero-shear viscosity $\eta_{0}$ of blends (closed circles) and narrow-distribution polystyrenes (open circles) plotted logarithmically against weightaverage molecular weight $\bar{M} .^{4}$

Polymer J., Vol. 1, No. 5, 1970 
A Constitutive Model for Molecular Weight and Concentration Effects in Polymer Blends

Table I. Summary of results for bimodal distribution of molecular weights in polymer blends (both $M_{i}^{*}<1$ or $M_{i}^{*}>1$ ) a,b

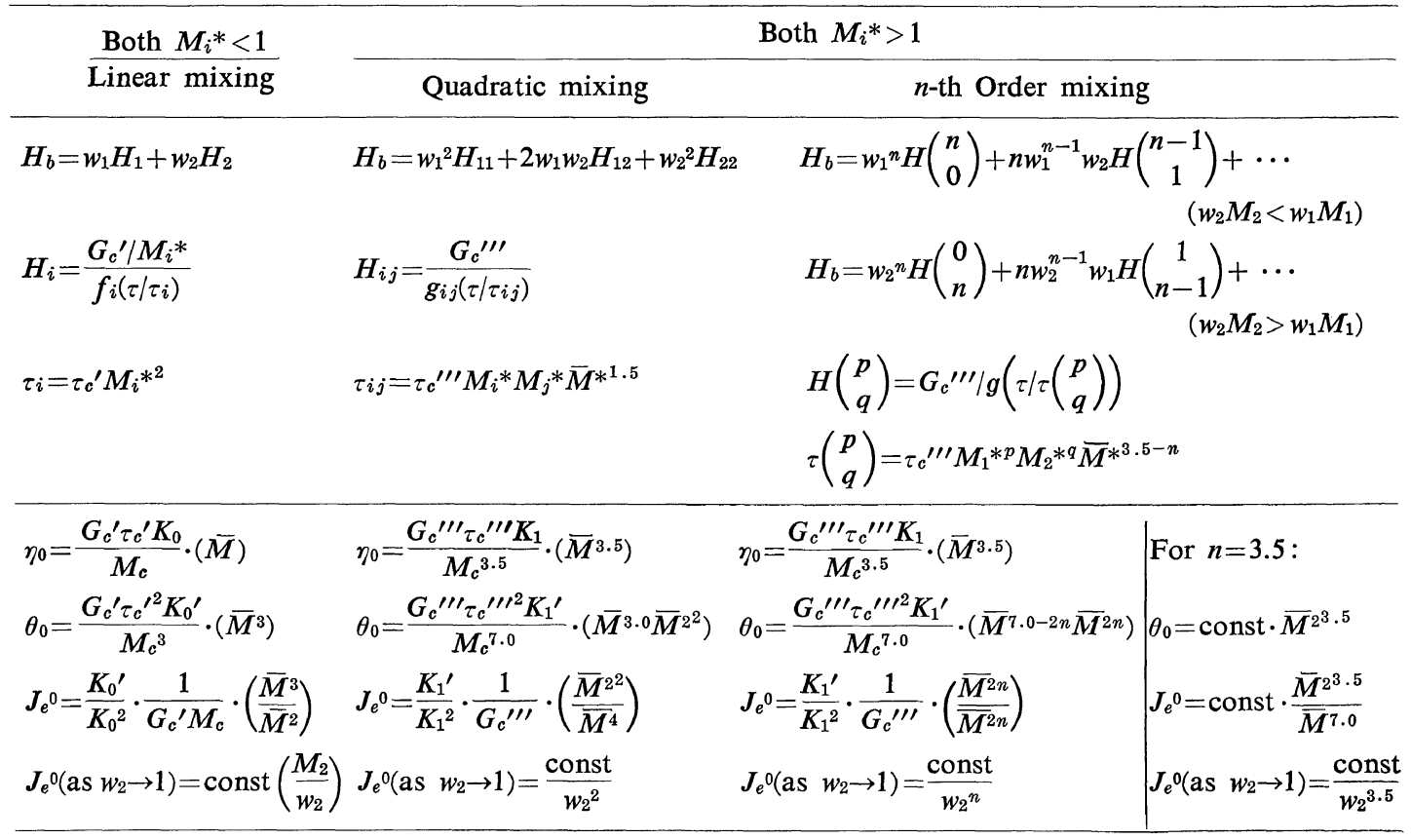

a $\bar{M}^{\alpha}=x_{1} M_{1}^{\alpha}+x_{2} M_{2} \alpha$

b Many of these results may be cast in terms of the " $z$-average" molecular weight by the relation $\bar{M}_{z}=\bar{M}^{2} / \bar{M}$.

Figure 1, which presents the data of Masuda, et al.

The corresponding results for the elastic coefficient are quite interesting. We write out here only the case for $M_{i}^{*}>1$.

$$
\begin{aligned}
\theta_{0}= & \frac{G_{c}{ }^{\prime \prime \prime} \tau_{c}{ }^{\prime \prime \prime}{ }^{\prime 2} K_{1}{ }^{\prime} \bar{M}^{3.0}}{M_{c}^{7.0}} \\
& \times\left\{w_{1}{ }^{2} M_{1}{ }^{4}+2 w_{1} w_{2} M_{1}{ }^{2} M_{2}{ }^{2}+w_{2}{ }^{2} M_{2}{ }^{4}\right\}
\end{aligned}
$$

where

$$
K_{1}^{\prime}=\int_{a_{11}}^{b_{11}} \frac{\xi d \xi}{g_{11}(\xi)}=\int_{a_{12}}^{b_{12}} \frac{\xi d \xi}{g_{12}(\xi)}=\int_{a_{22}}^{b_{22}} \frac{\xi d \xi}{g_{22}(\xi)}
$$

The \{ \} bracket in this case cannot be reduced simply to $\bar{M}$, but rather to $\bar{M}^{2}$, as defined in Table I. All of these results as well as the predictions for $J_{e}^{0}$ and for $n$-th order mixing laws, are summarized in Table I. In this latter case, " $n$ " is not necessarily an integer. One notes that for $M_{i}^{*}<1$ the viscosity is the extended Rouse theory. ${ }^{10}$ Results of the Zimm-type could be obtained by introducing $\tau_{i}=\tau_{c}{ }^{\prime} M_{i} *^{1.5}$ in eq 14.

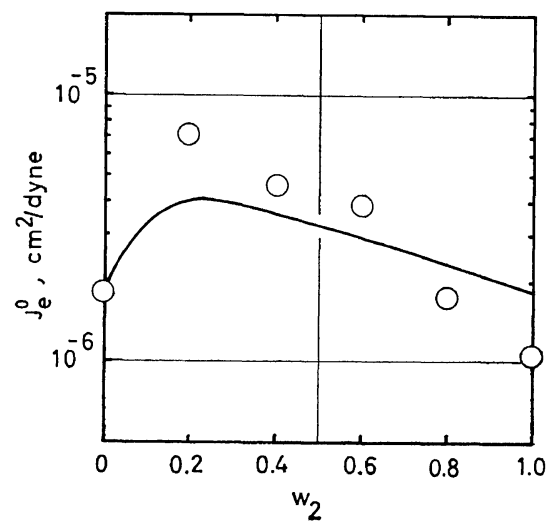

Figure 2. The steady-state compliance as a function of composition for a binary polystyrene blend. The solid line and open circles represent the theoretical (eq 25) and experimental ${ }^{4}$ results, respectively.

The predicted dependence of $J_{e}^{0}$ on composition for molecular weights above entanglement point is shown in Figure 2, again with the data 
of Masuda, et al. The plotted theoretical line in Figure 2 is

$$
\frac{\left.J_{e}^{0} \text { (blend }\right)}{J_{e}^{0}(\text { pure } 1)}=\frac{\left(w_{1} M_{1}^{2}+w_{2} M_{2}^{2}\right)^{2}}{\left(w_{1} M_{1}+w_{2} M_{2}\right)^{4}}
$$

where $M_{1}=46900$ and $M_{2}=167000$. The predicted form of $J_{e}^{0}$ seems to be in reasonable agreement with the data, considering the experimental variations in the data. Considering all of the data together, one expects $J_{e}^{0}$ (pure 1$)=J_{e}^{0}$ (pure 2) that is, equal values on the intercept. Also the form $J_{e}{ }^{0}=\mathrm{const} / w_{2}{ }^{2}$ for $w_{2} \rightarrow 1$ comes out of the theory, although this is not obvious in Figure 2. The situation for $M_{i}^{*}<1$ is not so clearly defined, as elastic coefficient data are apparently not available for narrow-distribution polymer blends.

The results for $n$-th order mixing are also shown in Table I. For $M_{i}^{*}>1$ it is tempting to set $n=3.5$, thereby placing all of the molecular weight dependence of the time constants in the factors $M_{1}$ and $M_{2}$. Possibly this will be necessary, but the available evidence for the compliance at $w_{2} \rightarrow 1$ seems to support a quadratic form or something near it. In developing the model further for multiple regimes, the quadratic form will be used.

\section{GENERALIZATION TO ALLOW FOR EFFECTS FROM MULTIPLE REGIMES}

While the previous results allow us to model either the transition regime or the terminal regime, they do not deal with cases where all regimes are important. We attack this more complicated problem by considering the sketches and equations in Figure 3, where $I$ is the transition regime, II is the rubbery plateau regime, and III is the terminal flow regime. Note that the choice $m=\infty$ reduces the latter equation to the familiar "box" distribution. ${ }^{19}$ The constants $G_{c}{ }^{\prime}, \tau_{c}{ }^{\prime}, G_{c}{ }^{\prime \prime}, G_{c}{ }^{\prime \prime \prime}$ and $\tau_{c}{ }^{\prime \prime \prime}$ are "universal constants" for a particular polymer, which we obtain from master curves for the various regimes after all molecular weight effects have been normalized out with the equations presented here. The essential feature in the model is that the various spectra $H_{11}, H_{12}$ and $H_{22}$ do not necessarily cross from one regime to the other at the same point, reflecting the fact that the normalization procedure for molecular weight is different in the various regimes. We handle this by solving for the junction time constants $\tau^{I}$ and $\tau_{i j}^{I I}$ as follows (both $M_{i}^{*}>1$ ):

At $\tau^{I}$ :

$$
\frac{G_{c}{ }^{\prime}}{\left(\tau^{I} / \tau_{c}{ }^{\prime}\right)^{1 / 2}}=G_{c}{ }^{\prime \prime}, \quad \text { or } \quad \tau^{I}=\tau_{c}{ }^{\prime}\left(\frac{G_{c}{ }^{\prime}}{G_{c}{ }^{\prime \prime}}\right)^{2}
$$

At $\tau_{i j}^{I I}$ :

$$
\begin{aligned}
G_{c}{ }^{\prime \prime} & =\frac{G_{c}{ }^{\prime \prime \prime}}{\left[\tau_{i j}^{I I} / \tau_{c}{ }^{\prime \prime \prime} M_{i}{ }^{*} M_{j}{ }^{*} \overline{M^{*}} \overline{1.5}\right]^{m}} \text { or } \\
\tau_{i j}^{I I} & =\tau_{c}{ }^{\prime \prime \prime}\left(\frac{G_{c}{ }^{\prime \prime \prime}}{{G_{c}}^{\prime \prime}}\right)^{1 / m} M_{i}{ }^{*} M_{j}{ }^{*} \bar{M}^{* 1.5}
\end{aligned}
$$

We also postulate

$$
\tau_{i j}^{\max }=b . . \tau_{c}^{\prime \prime \prime} M_{i} * M_{j} * \bar{M}^{* 1.5}
$$

where $b .$. is a constant. Note that $\tau^{I}$ does not depend on molecular weight while $\tau_{i j}^{I I}$ and $\tau_{i j}^{\max }$ depend significantly on molecular weight as well as the weight fractions. These results seem to be in accord with the data. ${ }^{4}$

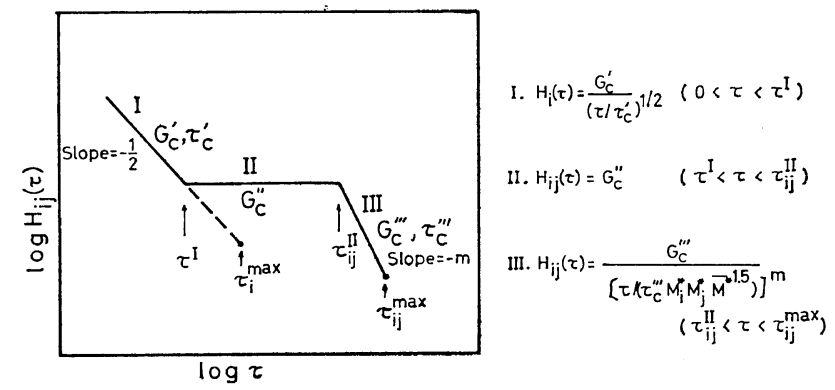

Figure 3. Sketch of assumed forms for the relaxation spectra in the various regimes. 
The corresponding results for $M_{i}^{*}<1$ includes just the transition (I) regime, using the equation given in Figure 3 for that regime but terminating at the following maximum relaxation time instead of at $\tau^{I}$.

$$
\tau_{i}^{\max }=b \cdot \tau_{c}^{\prime} M_{i}^{* 2} \quad\left(\text { both } M_{1}^{*} \text { and } M_{2}^{*}<1\right)
$$

where $b$. is a constant.

This, then, completely defines the spectra and allows us to insert the linear and quadratic mixing equations (eq 11 and 12) into the rheological equations (eq 2, 3, and 4). Omitting the steps which are lengthy but straightforward, one has finally:

For both $M_{1}^{*}$ and $M_{2}^{*}<1$ :

The results of the first column of Table I with $K_{0}=2 b .^{1 / 2}$ and $K_{0}{ }^{\prime}=2 / 3 b^{3 / 2}$

For both $M_{1}^{*}$ and $M_{2}^{*}>1$ :

$$
\begin{aligned}
& \eta_{0}=2 \frac{G_{c}{ }^{\prime 2}}{{G_{c}}^{\prime \prime}} \tau_{c}{ }^{\prime}+G_{c}{ }^{\prime \prime} \tau_{c}{ }^{\prime}\left\{A \overline{M^{*}}{ }^{3.5}\right. \\
& -B\}+G_{c}{ }^{\prime \prime \prime} \tau_{c}{ }^{\prime \prime \prime} \overline{M^{*}}{ }^{3.5}\left(\frac{b_{. .}^{1-m}-C^{1-m}}{1-m}\right)
\end{aligned}
$$

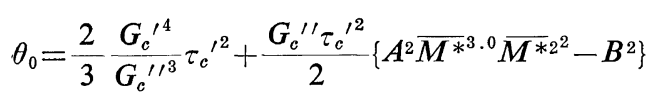

$$
\begin{aligned}
& +G_{c}{ }^{\prime \prime \prime} \tau_{c}{ }^{\prime \prime \prime} \bar{M}^{* 3.0} \overline{M^{*} 2^{2}}\left(\frac{b_{. \cdot}^{2-m}-C^{2-m}}{2-m}\right)
\end{aligned}
$$

where

$$
\begin{aligned}
& A=\frac{\tau_{c}{ }^{\prime \prime \prime}}{\tau_{c}{ }^{\prime}}\left(\frac{G_{c}{ }^{\prime \prime \prime}}{G_{c}{ }^{\prime \prime}}\right)^{1 / m}, \quad B=\left(\frac{G_{c}{ }^{\prime}}{G_{c}{ }^{\prime \prime}}\right)^{2}, \\
& C=\left(\frac{G_{c}{ }^{\prime \prime \prime}}{G_{c}{ }^{\prime \prime}}\right)^{1 / m}
\end{aligned}
$$

Eq 30 and 31 reduce to the quadratic results shown in Table I when the last term dominates but not when the first term dominates. This reflects the fact that in the previous work for $M^{*}<1$, it was assumed that the maximum relaxation time in the transition regime (when no plateau was present) had the same form as $\tau_{i}$ for that regime-i.e., was proportional to $M_{i}{ }^{2}$. In eq 30 and 31 the transition region is terminated by a plateau region which commences at certain values of $\tau^{I}$, which is independent of molecular weight.

In general, then, the various regimes mix in different ways as $H_{11}, H_{12}$ and $H_{22}$ for the various species overlap each other. A sketch of the

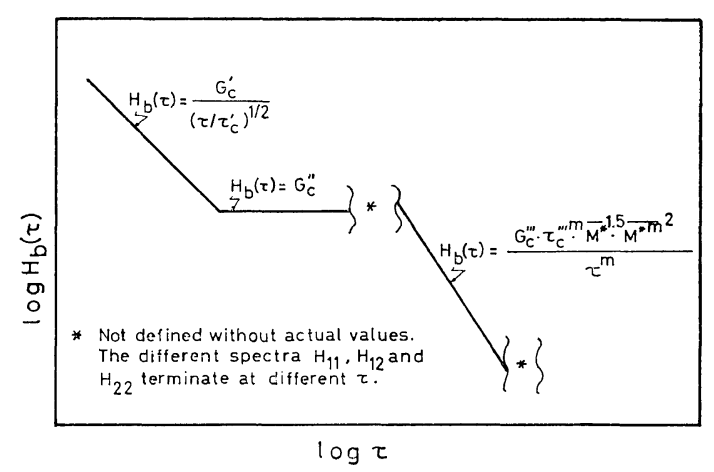

Figure 4. Sketch of predicted relaxation spectrum for a blend (both $M_{1}{ }^{*}$ and $M_{2}{ }^{*}>1$ ).

form of $H_{b}(\tau)$, so far as it can be given generally is shown in Figure 4. Because the $H_{11}, H_{12}$ and $H_{22}$ spectra terminate at different values of $\tau$, the junction regions may show complex forms.

\section{GENERALIZATION FOR ARBITRARY MOLECULAR WEIGHT MIXTURES}

The previous discussion requires either $M_{i}^{*}>1$ or $M_{i}^{*}<1$. We will now discuss the more complicated problem where one species (say 1) is below entanglement and the other (2) is above entanglement. It will probably not be possible to elucidate all of the details of this structure from experimental data but a reasonable physical picture can be imagined. We visualize a network of some kind if either $w_{1} M_{1}{ }^{*}$ or $w_{2} M_{2}{ }^{*}>$ 1. If both $w_{1} M_{1}{ }^{*}$ and $w_{2} M_{2}{ }^{*}>1$ we will have the prior results summarized in Figures 3 and 4 and eq 30-32. If only $w_{2} M_{2}{ }^{*}>1$ (but $w_{1} M_{1}{ }^{*}$ near or $<1$ ) we visualize a partial network consisting mostly of species 2 , with some of species 1 "associated" with the network, but possibly some of species 1 separated from it, depending on the molecular weight and concentration levels.

For species 2 it seems reasonable to take the short and long relaxation times as additive in the sense of the previous section. That is, a Rouse-like form can be used for short relaxation times and added to a network form for long relaxation times. This is in the spirit of the Blizard-Marvin model ${ }^{20}$ for short relaxation time response in a network and is associated with a physical picture in which the segments 
Table II. Generalization for arbitrary molecular weight mixtures

$$
\begin{aligned}
& \text { For } \tau<\tau^{I}: \quad H_{b}=w_{1} H_{1}+w_{2} H_{2} \text { : } \\
& H_{i}=\frac{G_{c}^{\prime} / M_{i}^{*}}{\left(\tau / \tau_{c}^{\prime} M_{i}^{* 2}\right)^{1 / 2}} \quad\left(\text { for } \quad 0<\tau<\tau^{I}\right) \\
& \text { For } \tau>\tau^{I}: \quad H_{b}=\left(1-f_{1}\right) w_{1} H_{1}+f_{1} w_{1}^{2} H_{11}+2 f_{1}{ }^{1 / 2} f_{2^{1 / 2}} w_{1} w_{2} H_{12}+f_{2} w_{2}^{2} H_{22}+\left(1-f_{2}\right) w_{2} H_{2} \\
& H_{i}=\frac{G_{c}^{\prime} / M_{i}^{*}}{\left(\tau / \tau_{c}^{\prime} M_{i}^{* 2}\right)^{1 / 2}} \\
& \text { (for } \left.\tau^{I}<\tau<\tau_{i} \text { max }\right) \\
& \tau_{i} \max =b . \tau_{c}^{\prime} M_{i}^{* 2} \\
& H_{i j}=G_{c}^{\prime \prime} \quad\left(\text { for } \tau^{I}<\tau<\tau_{i j}^{I I}\right) \\
& H_{i j}=\frac{G_{c}^{\prime \prime \prime}}{\left(\tau / \tau_{c} M_{i}^{*} M_{j}^{*} \bar{M}^{* 1.5}\right)^{m}} \quad\left(\text { for } \quad \tau_{i j}^{I I}<\tau<\tau_{i j}^{\max }\right. \\
& \tau_{i j}^{I I}=\tau_{c}^{\prime \prime \prime}\left(\frac{G_{c}^{\prime \prime \prime}}{G_{c}{ }^{\prime \prime}}\right)^{1 / m} M_{i} * M_{j} * \overline{M^{* 1.5}} \\
& \tau_{i j}^{\max }=b . . \tau_{c}^{\prime \prime \prime} M_{i}^{*} M_{j}^{*} \bar{M}^{* 1.5} \\
& f_{i}=f\left(w_{i} M_{i}\right)\left\{\begin{array}{l}
f_{i}=0 \text { if } w_{i} M_{i} \ll M_{c}\left(\text { or } M_{e}\right) \\
f_{i}=1 \text { if } w_{i} M_{i} \gg M_{c}\left(\text { or } M_{e}\right)
\end{array}\right.
\end{aligned}
$$

between the entanglement points behave independently of their presence in the network. But there is a different kind of concept involved when both species are contributing long time modes but by different mechanisms. We associate long relaxation times with long molecular segments and these long molecular segments are either involved in the network or are coiled up and separated from it, but not both. At any level of relaxation time, an element not involved in the network probably will have the Rousetype of spectrum.

As a framework for summarizing these ideas we propose the following phenomenological equation, good for long relaxation times:

$$
\begin{aligned}
H_{b}(\operatorname{long} \tau)= & \left(1-f_{1}\right) w_{1} H_{1}+f_{1} w_{1}{ }^{2} H_{11} \\
& +2 f_{1}^{1 / 2} f_{2}{ }^{1 / 2} w_{1} w_{2} H_{12}+f_{2} w_{2}{ }^{2} H_{22} \\
& +\left(1-f_{2}\right) w_{2} H_{2}
\end{aligned}
$$

where $f_{i}$ is an adjustable parameter to reflect the extent of participation in the network $\left(f_{i}=0\right.$ if no participation, and $f_{i}=1$ if full participation). This form reduces to the linear and quadratic mixing rules if $f_{i}=0$ and $f_{i}=1$, respectively. The factor $f_{i}$ must depend in some way on the molecular weights $M_{i}$, but there is a conceptual problem as to whether the relevant reference molecular weight is $M_{c}$ (the critical entanglement molecular weight) or $M_{e}$ (the molecular weight between junction points in the network). As one must imagine physically, these two values should be of the some magni- tude and seem to differ only by a factor of two or so. ${ }^{7}$ We will not attempt to model $f_{i}$ except to state its values in the limits.

The various assumptions can be summarized by the equations in Table II, which incorporates all of the results of the previous sections. Note that a number of different kinds of additivity rules may be read off depending on the molecular weights and concentrations of the two species.

While the discussion has been limited to blends of bulk polymers, the framework also has many of the necessary properties to explain polymer solution behavior. We think now of a system with component 1 as the solvent and component 2 as the polymer. In the case of solutions, however, there is a difficulty having to do with the interaction of the solvent with the polymer. In dilute solution theory the time constant $\tau_{c}{ }^{\prime}$ in eq 14 ( $\zeta_{0}$ in the Rouse theory) must be assumed proportional to the solvent viscosity. This must be so if the results are to be factored into the form

$$
\eta_{0}=\eta_{\text {solv }}\left[1+\text { const } \cdot c_{2} M_{2}\right]
$$

where $\eta_{\text {solv }}$ is the solvent viscosity and $c_{2}$ is the polymer coencentration. This form, of course, is the appropriate one in discussing intrinsic viscosity. In the case of undiluted polymers, however, the time constant $\tau_{c}{ }^{\prime}$ must be taken to be independent of the molecular weights and compositions (as in the present model). In a completely general model, then, one must deal 
A Constitutive Model for Molecular Weight and Concentration Effects in Polymer Blends

with the merger of the assumption about $\tau_{c}{ }^{\prime}$ between the extremes of dilute solutions and undiluted polymers. We do not attempt that here. But for polymer solutions of high concentration, we note that many of the strong dependences of the elastic compliance $J_{e}{ }^{0}$ on the concentrations and molecular weight can be read off of the results in Table II. In particular, one finds a dependence of the compliance on $c_{2}{ }^{-2}$ for high polymer concentrations, which compares with dependences ranging from $c_{2}{ }^{-2}$ to $c_{2}{ }^{-3} \cdot{ }^{21,22}$

\section{GENERALIZATION TO A WIDE DISTRI- BUTION OF MOLECULAR WEIGHTS}

The generalization of the long $\tau$ blending equation of Table II for a wide distribution of molecular weights is

$$
\begin{aligned}
H_{b}= & \left(1-f_{1}\right) w_{1} H_{1}+\left(1-f_{2}\right) w_{2} H_{2}+\left(1-f_{3}\right) w_{3} H_{3}+\cdots \\
& +f_{1} w_{1}{ }^{2} H_{11}+f_{2} w_{2}{ }^{2} H_{22}+f_{3} w_{3}{ }^{2} H_{33}+\cdots \\
& +2 f_{1}{ }^{1 / 2} f_{2}{ }^{1 / 2} w_{1} w_{2} H_{12}+2 f_{2}{ }^{1 / 2} f_{3}{ }^{1 / 2} w_{2} w_{3} H_{23} \\
& +2 f_{1}^{1 / 2} f_{3}{ }^{1 / 2} w_{1} w_{3} H_{13}+\cdots
\end{aligned}
$$

All of the equations presented for $H_{i}$ and $H_{i j}$ also apply for the multi-species (wide distribution) case, including the results in Tables I and II, except for the $n$-th order mixing case in Table I. This case is based on the binomial theorem and it is not clear how it can be generalized. The following definition for the averages would apply

$$
\begin{aligned}
\bar{M} & =\sum_{i=1}^{N} w_{i} M_{i}, & \overline{M^{*}} & =\sum_{i=1}^{N} w_{i} H_{i}{ }^{*} \\
\overline{M^{2}} & =\sum_{i=1}^{N} w_{i} M_{i}{ }^{2}, & \overline{M^{* 2}} & =\sum_{i=1}^{N} w_{i} M_{i} *^{2}
\end{aligned}
$$

where $N$ is the number of species.

An interesting result which could come from the wide distribution equations is a considerable overlap between the various regimes. Because the rubbery plateaus for the different species terminate at different values of $\tau$, it is possible for the rubbery region of the high molecular weight species to overlap into the terminal flow region of the other species, thereby modifying the observed slope there. A reduction of the slope for wide distribution mixtures has been observed experimentally. ${ }^{4}$
Acknowledgements. This study was supported by a grant for scientific research (Kagaku Kenkyūhi) from the Ministry of Education. The first-named author (DCB) acknowledges with thanks the support of the National Science Foundation (under its U.S.-Japan Cooperative Science Program) for an eight-month research visit at Kyoto University.

\section{REFERENCES}

1. R. S. Porter and J. F. Johnson, Chem. Rev., 66, 1 (1966).

2. G. C. Berry and T. G. Fox, Adv. Polym. Sci., 5, 261 (1968).

3. G. Akovali, J. Polym. Sci., Part A-2, 5, 875 (1967).

4. T. Masuda, K. Kitagawa, T. Inoue, and S. Onogi, Macromolecules, 3, 116 (1970).

5. S. Onogi, T. Masuda, and K. Kitagawa, Macromolecules, 3, 109 (1970).

6. D. C. Bogue and J. O. Doughty, Ind. Eng. Chem. Fundamentals, 5, 243 (1966).

7. J. D. Ferry, "Viscoelastic Properties of Polymers," John Wiley \& Sons, Inc., New York, N.Y., 1961; ibid., 2nd ed., 1970.

8. H. Leaderman, "Elastic and Creep Properties of Filamentous Materials and Other High Polymers," The Textile Foundation, Washington, 1943.

9. P. E. Rouse, J. Chem. Phys., 21, 1272 (1953).

10. J. D. Ferry, R. F. Landel, and M. L. Williams, J. Appl. Phys., 26, 104 (1955).

11. B. H. Zimm, J. Chem. Phys., 24, 269 (1956).

12. A. S. Lodge, "Elastic Liquids," Academic Press, New York, N.Y., 1964.

13. M. Yamamoto, J. Phys. Soc. Japan, 11, 413 (1956); 12, 1149 (1957); 13, 1200 (1958).

14. S. Hayashi, J. Phys. Soc. Japan, 18, 131, 249 (1963); 19, 101, 2306 (1964).

15. See, for example, A. V. Tobolsky, J. J. Aklonis, and G. Akovali, J. Chem. Phys., 42, 723 (1965); T. Fujimoto, N. Ozaki, and M. Nagasawa, J. Polym. Sci., Part A-2, 6, 129 (1968).

16. K. Ninomiya, J. Colloid Sci., 14, 49 (1959).

17. K. Ninomiya and J. D. Ferry, J. Colloid Sci., 18, 421 (1963).

18. M. C. Williams, AIChE Journal, 12, 1064 (1966).

19. A. V. Tobolsky, "Properties and Structure of Polymers," John Wiley \& Sons, Inc., New York N.Y., 1963, Chapter 3.

20. (a) R. B. Blizard, J. Appl. Phys., 22, 730 (1951); (b) R. S. Marvin and H. Oser, J. Res. NBS, 
D. C. Bogue, T. Masuda, Y. Einaga, and S. Onogi

66B, 171 (1962).

21. (a) T. Kotaka and K. Osaki, J. Polym. Sci., Part C, 15, 453 (1966); (b) W. W. Graessley and L. Segal, Macromolecules, 2, 49 (1969); (c)
T. Masuda, N. Toda, Y. Aoto, and S. Onogi, to be submitted to this journal.

22. Y. Einaga, K. Osaki, M. Kurata, and M. Tamura, Macromolecules, to be published. 